\title{
Slip Flow due to a Stretching Cylinder
}

\author{
C.Y. Wang ${ }^{1}$ and Chiu-On $\mathrm{Ng}^{2}$ \\ ${ }^{1}$ Department of Mathematics, Michigan State University, East Lansing, MI, USA \\ ${ }^{2}$ Department of Mechanical Engineering, University of Hong Kong, \\ Pokfulam Road, Hong Kong
}

\begin{abstract}
The slip flow due to a stretching cylinder is studied. A similarity transform reduces the Navier-Stokes equations to a set of nonlinear ordinary differential equations. Asymptotic solutions for large Reynolds number and small slip show the problem can be related to the existing two-dimensional stretching cases. Due to algebraic decay, the equations are further transformed through a compressed variable, and then integrated numerically. It is found that slip greatly reduces the magnitudes of the velocities and the shear stress.
\end{abstract}

Keywords: Slip flow; Stretching Cylinder; Similarity

\section{Introduction}

The viscous flow due to a stretching boundary occurs in the extrusion of metal, plastic and food products. The stretching causes the entrainment of the adjacent fluid, which in turn affects the resistance and the solidification of the extruded material. If the extrusion velocity is linear with respect to distance [1], which usually occurs with material subjected to constant strain, similarity solutions of the fluid problem may exist [2]. The two-dimensional stretching of a flat sheet was solved by Crane [3] and the axisymmetric radial stretching of a surface by Wang [4]. Brady and Acrivos [5] considered the flow inside a stretching channel or tube, while Wang [6] studied the flow outside a stretching cylinder. The above sources, and their many extensions, applied the no-slip boundary condition between the fluid and the extrusate.

In certain cases, the no-slip condition does not hold and should be replaced by a partial slip condition. These occur when the fluid is a rarefied gas [7], or when it is particulate such as blood, foam, emulsion or suspension [8]. Slip also occurs on hydrophobic surfaces, especially in micro- and nano-fluidics [9]. 
Two- dimensional stretching surface with partial slip was studied by Andersson [10] and Wang [11], and the axisymmetric case by Ariel [12]. It was found that slip affects the velocities and the fluid resistances considerably. The purpose of the present paper is to investigate the axisymmetric similarity solution due to a stretching of a cylinder with a partial slip boundary. The results are relevant to the extrusion of filaments which are micron size and/or in a rarefied environment. It is also an exact similarity solution of the Navier-Stokes equations.

\section{Formulation}

Fig. 1 shows a cylinder of radius $a$ being stretched longitudinally with a surface velocity of $W s=2 b z$ where $b$ is a constant and $z$ is the axial direction. In addition, there is a longitudinal free stream velocity of $W$ at infinity. We use a transformation similar to that of Wang [6]

$$
u=-a b \frac{f(\eta)}{\sqrt{\eta}}, w=2 b z f^{\prime}(\eta)+W g(\eta), \quad \eta=\left(\frac{r}{a}\right)^{2}
$$

Here $(u, w)$ are velocities in the $(r, z)$ directions respectively and continuity is satisfied. Since there is no longitudinal pressure gradient, using Eq. (1), the Navier-Stokes equations reduce to the nonlinear ordinary differential equations

$$
\begin{aligned}
& \eta f^{\prime \prime \prime}(\eta)+f^{\prime \prime}=R\left[\left(f^{\prime}\right)^{2}-f f^{\prime \prime}\right] \\
& \eta g^{\prime \prime}(\eta)+g^{\prime}=R\left(g f^{\prime}-f g^{\prime}\right)
\end{aligned}
$$

where $R=b a^{2} / 2 v$ ( $v$ being the kinematic viscosity) is the Reynolds number.

On the cylinder surface, the partial slip condition [13] states that the slip velocity $u_{s}$ is proportional to the local shear stress $\tau$

$$
u_{s}=N \tau
$$

where $N$ is the slip coefficient. Eqs. $(1,4)$ then yield

$$
\begin{aligned}
& f^{\prime}(1)-1=\lambda f^{\prime \prime}(1) \\
& g(1)=\lambda g^{\prime}(1)
\end{aligned}
$$


Here $\lambda=2 N \rho v / a$ ( $\rho$ being the fluid density) is the normalized slip factor. Zero radial velocity on the surface requires

$$
f(1)=0
$$

And at infinity we have uniform flow $W$

$$
\begin{aligned}
& f^{\prime}(\infty)=0 \\
& g(\infty)=1
\end{aligned}
$$

For given $R$ and $\lambda$, Eqs. (2, 3, 5-9) are to be solved. Eqs. (2, 5) reduces to the no slip case of $[6]$ only when $\lambda=0$. Eqs. $(3,6)$ representing axial convection are entirely new.

\section{Asymptotic solution for large $R$ and small slip}

At large $R$ a boundary layer exists on the cylinder. The cylinder curvature is thus unimportant and the flow can be related to that on a two-dimensional stretching flat surface. Let $R>>1$ and $\lambda=\Lambda / \sqrt{R}<<1$. We expand as follows

$$
f=\frac{1}{\sqrt{R}} h(\xi)+O\left(R^{-1}\right), \quad g=k(\xi)+O\left(R^{-1 / 2}\right)
$$

where

$$
\xi=\sqrt{R}(\eta-1)
$$

is the boundary layer coordinate. Eqs. $(2,5,7,8)$ give

$$
\begin{aligned}
& \left(h^{\prime}\right)^{2}-h h^{\prime \prime}=h^{\prime \prime \prime} \\
& h^{\prime}(0)-1=\Lambda h^{\prime \prime}(0), \quad h(0)=0, \quad h^{\prime}(\infty)=0
\end{aligned}
$$

Eqs. $(12,13)$ represent the slip flow over a two dimensional stretching sheet. The solution was given by Andersson [10]

$$
h=1-c e^{-\xi}, \quad c=1 /(1+\Lambda)
$$

Eqs. $(3,6,9)$ yield

$$
k^{\prime \prime}+\left(1-c e^{-\xi}\right) k^{\prime}+c e^{-\xi} k=0, \quad k(0)=\Lambda k^{\prime}(0), \quad k(\infty)=1
$$


To solve Eq. (15), let

$$
K(s)=k(\xi), \quad s=e^{-\xi}
$$

Eq. (15) then becomes

$$
s K^{\prime \prime}+c s K^{\prime}-c K=0
$$

with the boundary conditions

$$
K(0)=1, \quad K(1)=-\Lambda K^{\prime}(1)
$$

The general solution to Eq. (17) is

$$
K=c_{1} s+c_{2} s \int \frac{e^{-c s}}{s^{2}} d s=c_{1} s-c_{2}\left[e^{-c s}-c s E(c s)\right]
$$

where

$$
E(z)=\int_{z}^{\infty} \frac{e^{-z}}{z} d z
$$

is the exponential integral. The boundary conditions give

$$
c_{2}=1, \quad c_{1}=-e^{-c}+E(c)
$$

Thus

$$
k=\exp \left(-c e^{-\xi}\right)+e^{-\xi}\left[E(c)-c E\left(c e^{-\xi}\right)-e^{-c}\right]
$$

Note that for no slip, $\Lambda=0, c=1$ and Eq. (22) reduces to the solution of the no slip flow over a stretching sheet found by Danberg and Fansler [14].

\section{Algebraic decay at large distances}

For large $\eta$, from Eqs. $(8,9)$ we can set

$$
f \sim c+\phi(\eta), \quad g \sim 1+\psi(\eta)
$$

where $c=f(\infty)$ is a constant and $\phi, \psi$ decay to zero as $\eta \rightarrow \infty$. For large enough $\eta$, Eqs. $(2,3)$ linearize to

$$
\eta \phi^{\prime \prime}+(1+R c) \phi^{\prime \prime}=0
$$




$$
\eta \psi^{\prime \prime}+(1+R c) \psi^{\prime}=R \phi^{\prime}
$$

The decay solutions to Eqs. $(24,25)$ are

$$
\phi=c_{1} \eta^{1-R c}, \quad \psi=c_{2} \eta^{-R c}+c_{1} R \eta^{1-R c}
$$

where $c_{1}, c_{2}$ are constants. We see that the functions decay algebraically (instead of exponentially as in stretching flat surfaces). Also, a necessary condition for existence of a solution is $c>1 / R$.

\section{Numerical method}

Due to the algebraic decay, the domain is too large for direct numerical integration of Eqs. $(2,3)$. We greatly compress the domain by an exponential transform

$$
\eta=e^{x}
$$

After some work, Eqs. (2, 3) become

$$
\begin{aligned}
& f_{x x x}-2 f_{x x}+f_{x}+R\left(f f_{x x}-f f_{x}-f_{x}^{2}\right)=0 \\
& g_{x x}+R\left(f g_{x}-f_{x} g\right)=0
\end{aligned}
$$

The boundary conditions (in $x$ ) are

$$
\begin{aligned}
& f(0)=0, \quad(1+\lambda) f_{x}(0)=1+\lambda f_{x x}(0) \\
& f_{x}(\infty)=0 \\
& g(0)=\lambda g_{x}(0) \\
& g(\infty)=1
\end{aligned}
$$

Given $R$ and $\lambda$, we guess $f_{x x}(0)$ and from Eq. (30) obtain $f(0)$ and $f_{x}(0)$. Then integrate Eq. (28) as an initial value problem by a standard Runge-Kutta algorithm. At large $\eta$ (about 15) we check whether $f_{x}$ decays to zero. Using shooting, the initial values can be accurately obtained. After $f$ is determined, Eq. (29) is similarly integrated with a guessed $g_{x}(0)$ and $g(0)$ from Eq. (32). A solution is obtained when $g-1$ decays to zero at large enough $x$. Table 1 shows the results. 
Table 1(a) Initial and final values for $R=0.1$

\begin{tabular}{|l|l|l|l|l|l|}
\hline$\lambda$ & $f_{x x}(0)$ & $f_{x}(0)=f^{\prime}(1)$ & $f^{\prime \prime}(1)$ & $c=f(\infty)$ & $g_{x}(0)=g^{\prime}(1)$ \\
\hline 0 & 0.518195 & 1 & -0.4818 & 15.061 & 0.1030 \\
\hline 0.1 & 0.501976 & 0.9547 & -0.4528 & 15.037 & 0.0965 \\
\hline 0.5 & 0.449207 & 0.8164 & -0.3672 & 14.984 & 0.0773 \\
\hline 1 & 0.401035 & 0.7005 & -0.2995 & 14.937 & 0.0621 \\
\hline 2 & 0.336058 & 0.5574 & -0.2213 & 14.879 & 0.0448 \\
\hline 5 & 0.237711 & 0.3648 & -0.1271 & 14.815 & 0.0250 \\
\hline 10 & 0.168397 & 0.2440 & -0.0756 & 14.769 & 0.0145 \\
\hline
\end{tabular}

Table 1(b) Initial and final values for $R=1$

\begin{tabular}{|l|l|l|l|l|l|}
\hline$\lambda$ & $f_{x x}(0)$ & $f_{x}(0)=f^{\prime}(1)$ & $f^{\prime \prime}(1)$ & $c=f(\infty)$ & $g_{x}(0)=g^{\prime}(1)$ \\
\hline 0 & -0.177752 & 1 & -1.1778 & 1.8786 & 0.3379 \\
\hline 0.1 & -0.112773 & 0.8988 & -1.0116 & 1.8401 & 0.2863 \\
\hline 0.5 & 0.0042342 & 0.6681 & -0.6638 & 1.7463 & 0.1815 \\
\hline 1 & 0.0522636 & 0.5261 & -0.4739 & 1.6862 & 0.1256 \\
\hline 2 & 0.0790586 & 0.3860 & -0.3070 & 1.6280 & 0.0777 \\
\hline 5 & 0.0807401 & 0.2340 & -0.1532 & 1.5585 & 0.0365 \\
\hline 10 & 0.0671050 & 0.1519 & -0.0848 & 1.5278 & 0.0190 \\
\hline
\end{tabular}

Table 1(c) Initial and final values for $R=10$

\begin{tabular}{|l|l|l|l|l|l|}
\hline$\lambda$ & $f_{x x}(0)$ & $f_{x}(0)=f^{\prime}(1)$ & $f^{\prime \prime}(1)$ & $c=f(\infty)$ & $g_{x}(0)=g^{\prime}(1)$ \\
\hline 0 & -2.344457 & 1 & -3.3445 & 0.3858 & 1.1402 \\
\hline 0.1 & -1.512162 & 0.7716 & -2.2838 & 0.3486 & 0.7700 \\
\hline 0.5 & -0.614427 & 0.4619 & -1.0763 & 0.2886 & 0.3532 \\
\hline 1 & -0.334432 & 0.3328 & -0.6672 & 0.2584 & 0.2143 \\
\hline 2 & -0.157647 & 0.2282 & -0.3859 & 0.2302 & 0.1204 \\
\hline 5 & -0.042532 & 0.1312 & -0.1738 & 0.1992 & 0.0514 \\
\hline 10 & -0.007778 & 0.0838 & -0.0916 & 0.1818 & 0.0258 \\
\hline
\end{tabular}

Table 1(d) Initial and final values for $R=100$

\begin{tabular}{|l|l|l|l|l|l|}
\hline$\lambda$ & $f_{x x}(0)$ & $f_{x}(0)=f^{\prime}(1)$ & $f^{\prime \prime}(1)$ & $c=f(\infty)$ & $g_{x}(0)=g^{\prime}(1)$ \\
\hline 0 & -9.183421 & 1 & -10.183 & 0.1064 & 3.661 \\
\hline 0.1 & -3.785225 & 0.5650 & -4.3502 & 0.0816 & 1.551 \\
\hline 0.5 & -1.187700 & 0.2708 & -1.4585 & 0.0587 & 0.5126 \\
\hline 1 & -0.633809 & 0.1831 & -0.8169 & 0.0495 & 0.2843 \\
\hline 2 & -0.319381 & 0.1204 & -0.4398 & 0.0416 & 0.1570 \\
\hline 5 & -0.119319 & 0.0672 & -0.1866 & 0.0331 & 0.0625 \\
\hline 10 & -0.053108 & 0.0426 & -0.0957 & 0.0281 & 0.0313 \\
\hline
\end{tabular}

We see $c>1 / R$ as predicted in the previous section. 


\section{Discussions}

Fig. 2 shows the similarity profile $f(\eta) / \sqrt{\eta}=f / \sqrt{e^{x}}$ for the radial velocity. Since the decay is too slow in $\eta$, we plotted the velocity against the compressed variable $x$. Because the radial velocity is zero on the surface and at infinity, there exists a maximum in the fluid. In contrast, for the two dimensional case the maximum is at infinity. The effect of slip is to decrease the velocity magnitude. Fig. 3 shows the similarity profile for the axial velocity $f^{\prime}(\eta)=f_{x}(x)$ which is due to stretching of the cylinder. Fig. 4 shows the function $g$, affected by $f$, which is due to the axial convection at infinity. Both contribute to the axial velocity $w$.

The shear stress on the surface is

$$
\tau_{r z}=\left.\mu \frac{\partial w}{\partial r}\right|_{r=a}=\frac{2 \mu}{a}\left[2 b z f^{\prime \prime}(1)+W g^{\prime}(1)\right]
$$

where the initial values are given in Table 1 . The location of zero shear is at

$$
z=\frac{-W g^{\prime}(1)}{2 b f^{\prime \prime}(1)}
$$

This location is positive on the $z$ axis if $W$ is positive, and negative if $W$ is negative. From Eq. (34) one can compute the total fluid dynamic drag for any segment of the stretching cylinder. Since both $\left|f^{\prime \prime}(1)\right|$ and $g^{\prime}(1)$ decrease with increased slip, the magnitude of drag would also decrease with increased slip.

How large is the slip factor $\lambda$ ? For rarefied gasses in micron sized tubes, $\lambda$ is of order 0.01. For fluids in superhydrophobic microtubes [15], $\lambda$ could be order of 1 . For lubricated surfaces, $\lambda$ could reach 100 [16].

\section{Acknowledgments}

The work was supported by the Research Grants Council of the Hong Kong Special Administrative Region, China, through Project No. HKU 715609E. 


\section{References}

[1] J. Vleggaar, Laminar boundary layer behavior on continuous, accelerating surfaces, Chem. Eng. Sci. 32 (1977) 1517-1525.

[2] C.Y. Wang, Exact solutions of the steady-state Navier-Stokes equations, Ann. Rev. Fluid Mech. 23 (1991) 159-177.

[3] L.J. Crane, Flow past a stretching plate, A. Angew. Math. Phys. 21 (1970) 645-647.

[4] C.Y. Wang, The three-dimensional flow due to a stretching flat surface, Phys. Fluids 27 (1984) 1915-1917.

[5] J.F. Brady and A. Acrivos, Steady flow in a channel or tube with an accelerating surface velocity, J. Fluid Mech. 112 (1981) 127-150.

[6] C.Y. Wang, Fluid flow due to a stretching cylinder, Phys. Fluids 31 (1988) 466468.

[7] F. Sharipov, and V. Seleznev, Data on internal rarefied gas flows, J. Phys. Chem. Ref. Data 27 (1998) 857-706.

[8] A. Yoshimura and R.K. Prudhomme, Wall slip corrections for Couette and parallel flow viscometers, J. Rheol. 32 (1988) 53-67.

[9] J. Eijkel, Liquid slip in micro- and nanofluidics: recent research and its possible implications, Lab on Chip 7 (2007) 299-301.

[10] H.I. Andersson, Slip flow past a stretching surface, Acta Mech. 158 (2002) 121125.

[11] C.Y. Wang, Flow due to a stretching boundary with partial slip - an exact solution of the Navier-Stokes equations, Chem. Eng. Sci. (2002) 3745-3747.

[12] P.D. Ariel, Generalized three dimensional flow due to a stretching sheet, Ziet. Angew. Math. Mech. 83 (2003) 844-852.

[13] C.L.M. Navier, Sur les Lois du Mouvement des Fluides, C.R. Acad. Sci. 6 (1827) 389-440. 
[14] J.E. Danberg and K.S. Fansler, A non-similar moving wall boundary layer problem, Quart. Appl. Math. 34 (1976) 305-309.

[15] C.H. Choi and C.J. Kim, Large slip of aqueous liquid flow over a nanoengineered superhydrophobic surface. Phys. Rev. Lett. 96 (2006) \#066001.

[16] Wang, C.Y., 2007, "Stagnation Flow on a Cylinder with Partial Slip - An Exact Solution of the Navier-Stokes Equations,” IMA J. Appl. Math. 72, pp. 271-277.

\section{Figure Captions}

Fig. 1 The stretching cylinder with surface velocity $W s$ and a uniform flow $W$ at infinity.

Fig. 2 The radial velocity $f(\eta) / \sqrt{\eta}=f / \sqrt{e^{x}}$. From top: $\lambda=0,1,10$. (a) $R=1$ (b) $R=10$.

Fig. 3 The axial velocity $f^{\prime}(\eta)=f_{x}(x)$. From top: $\lambda=0,1,10$. (a) $R=1$ (b) $R=10$.

Fig. 4 The axial velocity $g$. From top for small $x: \lambda=0,1,10$. (a) $R=1$ (b) $R=10$. 


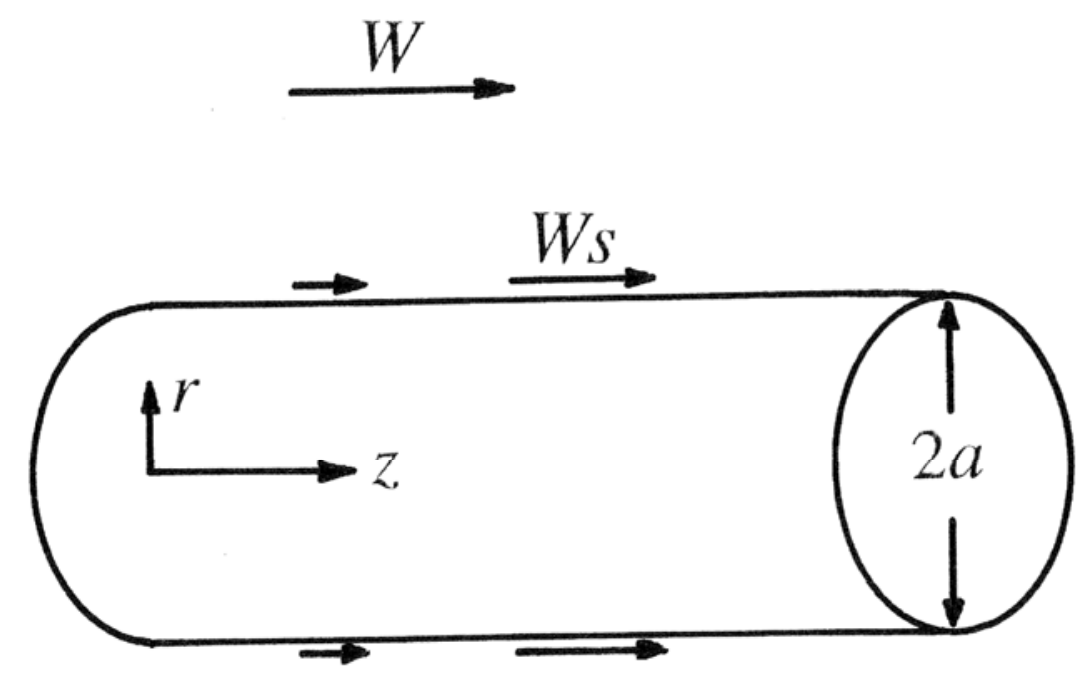

Figure 1 


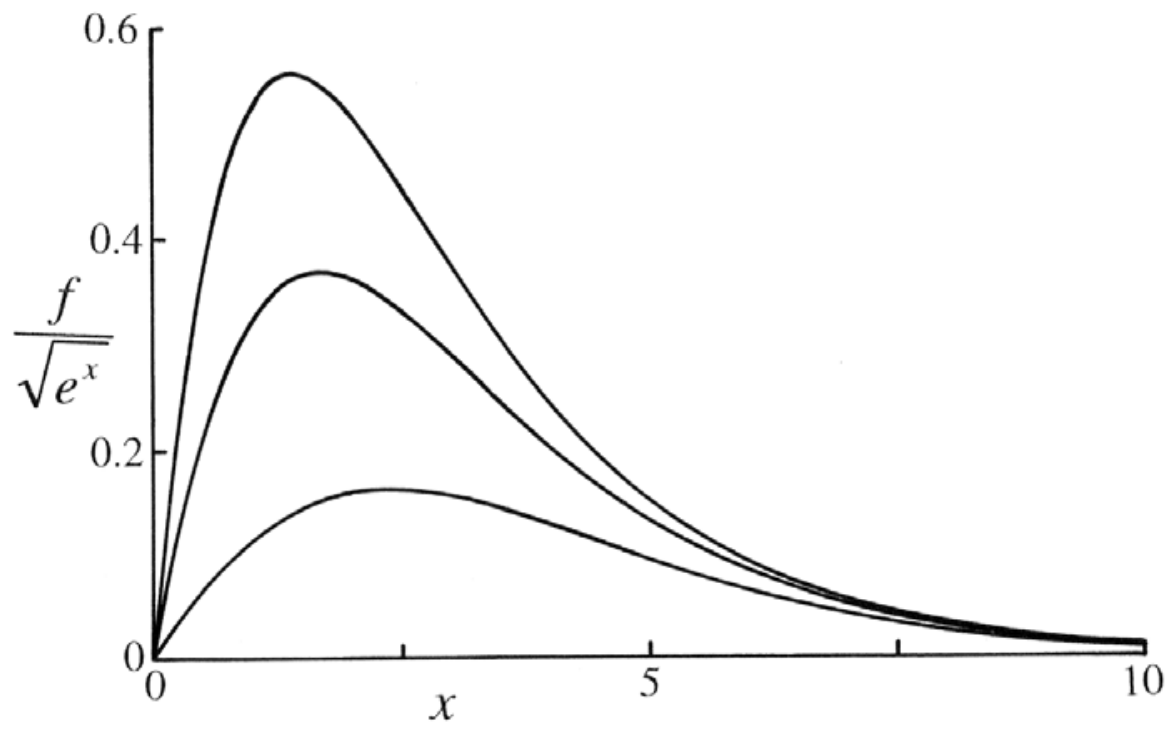

(a)

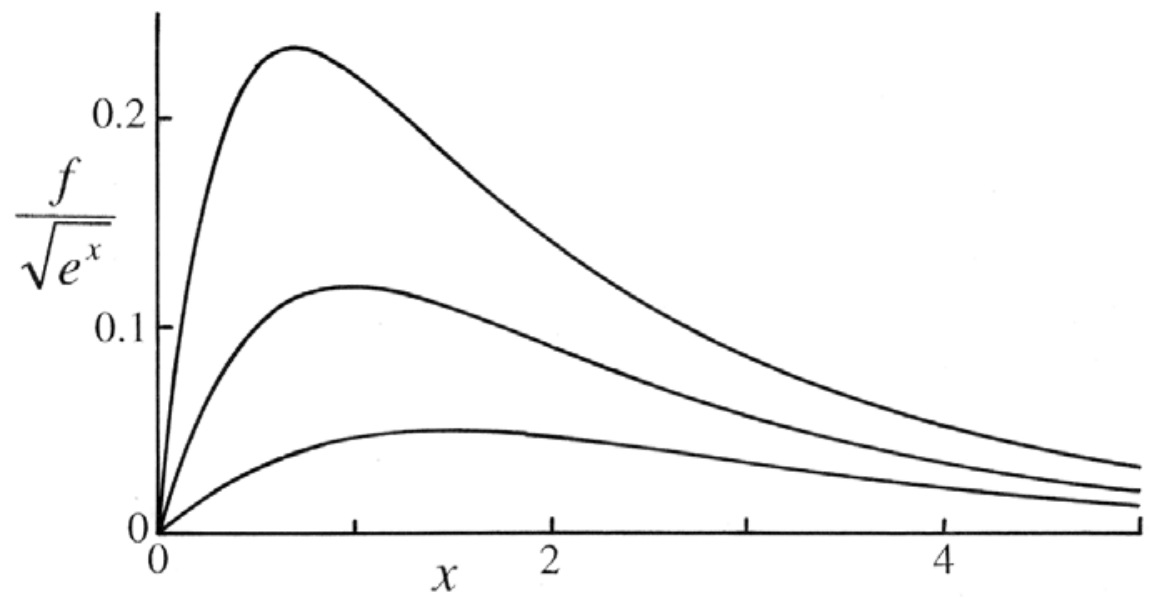

(b)

Figure 2 


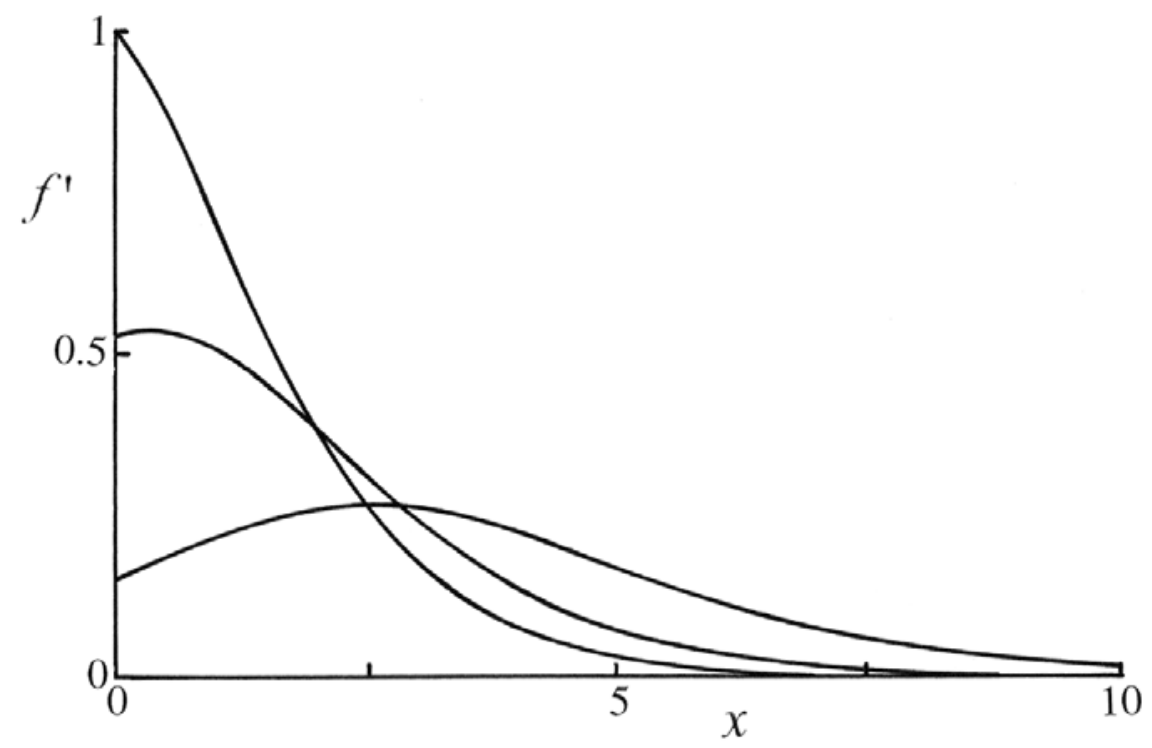

(a)

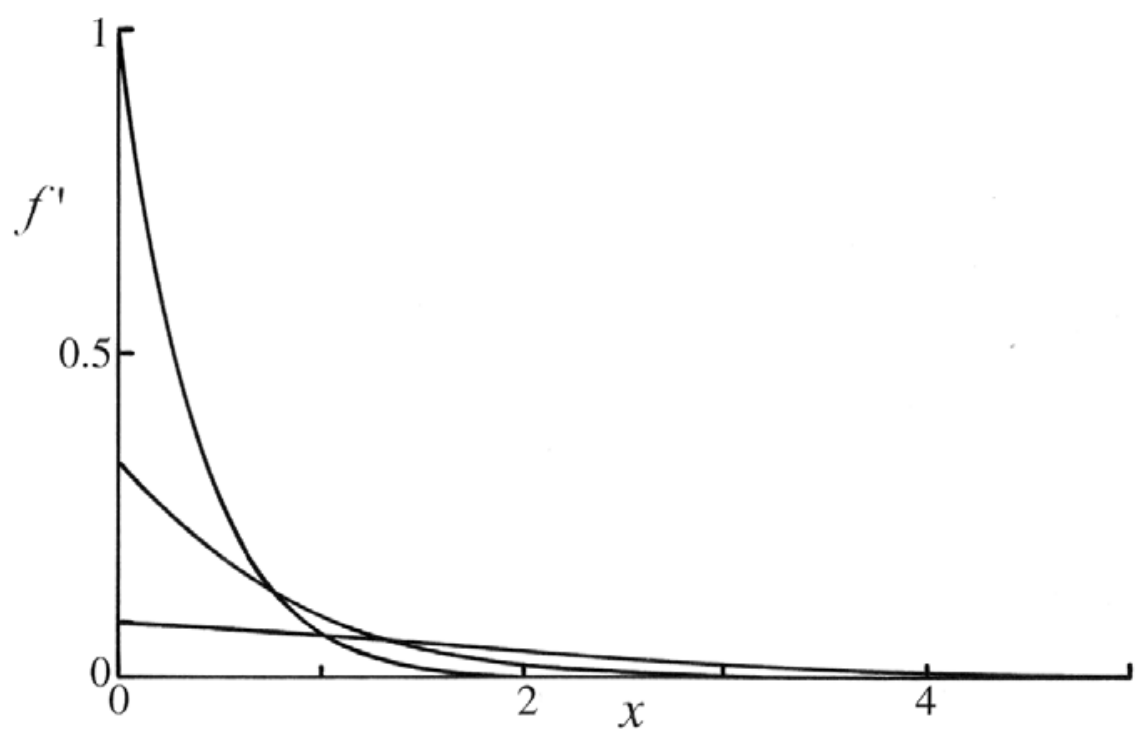

(b)

Figure 3 


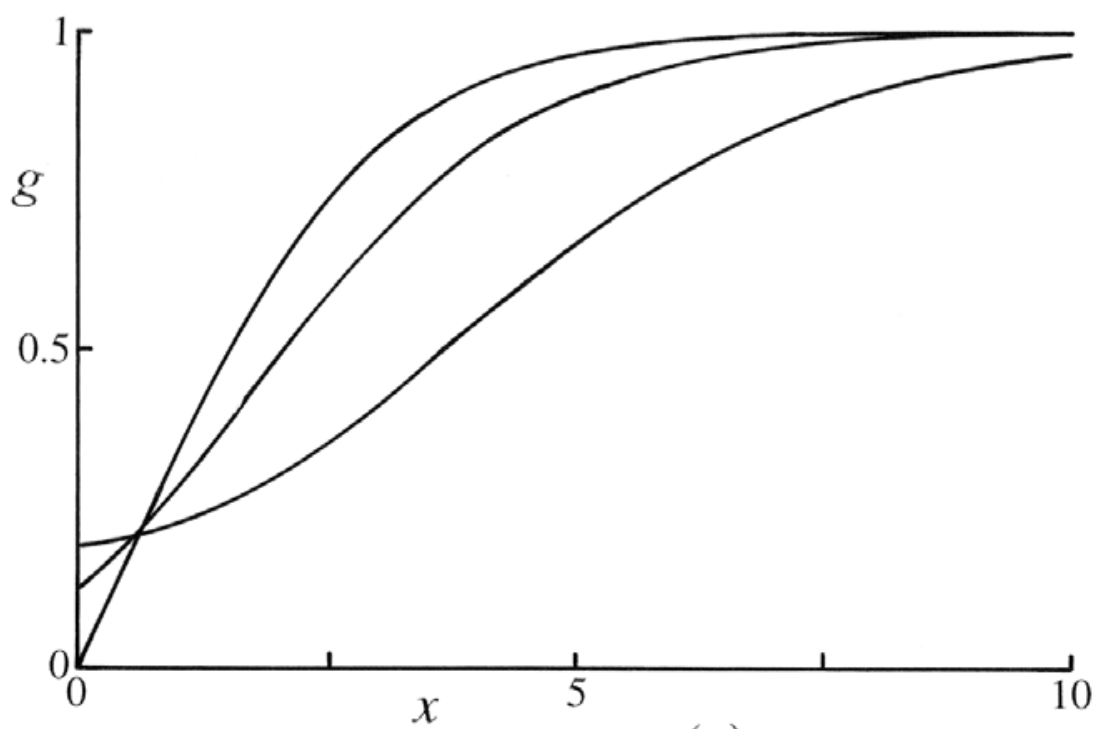

(a)

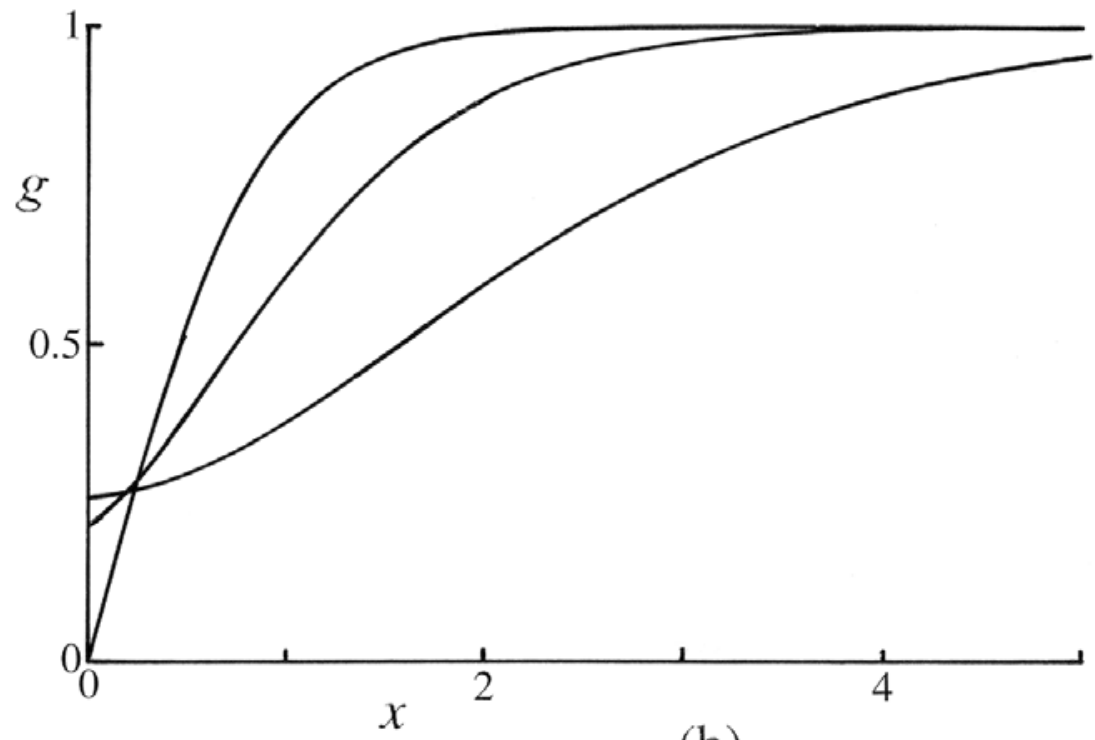

(b)

Figure 4 